\title{
EXTREME DISPERSIONS OF SEMICOHERENT AND MIXED SYSTEM LIFETIMES
}

\author{
PATRYK MIZIUŁA, ${ }^{*}$ Nicolaus Copernicus University \\ TOMASZ RYCHLIK, ${ }^{* *}$ Polish Academy of Sciences
}

\begin{abstract}
We consider semicoherent and mixed systems with exchangeable components. We present sharp lower and upper bounds on various dispersion measures (in particular, variance and median absolute deviation) of the system lifetime, expressed in terms of the system signature and the dispersion of a single component lifetime. We construct joint exchangeable distributions of component lifetimes with two-point marginals which attain the bounds in the limit.

Keywords: Semicoherent system; mixed system; Samaniego signature; exchangeable random variables; sharp bound; dispersion measure; variance; median absolute deviation

2010 Mathematics Subject Classification: Primary 62N05
\end{abstract}

Secondary 60E15; 62G30

\section{Introduction and results}

Semicoherent systems are basic notions of the reliability theory (see, e.g. Barlow and Proschan (1966), (1975)). They work if certain subsets of their components do too. These relations are precisely described by means of system structure functions $\phi:\{0,1\}^{n} \rightarrow\{0,1\}$, where $n$ denotes the number of components, $x_{i}=0$ and $x_{i}=1, i=1, \ldots, n$, mean that the $i$ th component works and does not work, respectively, and $\phi\left(x_{1}, \ldots, x_{n}\right)$ defines the working status of the system. A system is called semicoherent if $\phi(1, \ldots, 1)=1$, $\phi(0, \ldots, 0)=0$, and $\phi\left(x_{1}, \ldots, x_{n}\right) \geq \phi\left(y_{1}, \ldots, y_{n}\right)$ when $x_{i} \geq y_{i}, i=1, \ldots, n$. These conditions mean that the system with all working (respectively, failed) elements operates (respectively, does not operate), and that failure of some components cannot result in repairing a failed system. A system is called coherent if it does not contain irrelevant components. Formally, it means that, for every $i=1, \ldots, n$ there exists $x_{1}, \ldots, x_{i-1}, x_{i+1}, \ldots, x_{n} \in\{0,1\}$ such that $\phi\left(x_{1}, \ldots, x_{i-1}, 1, x_{i+1}, \ldots, x_{n}\right)-\phi\left(x_{1}, \ldots, x_{i-1}, 0, x_{i+1}, \ldots, x_{n}\right)=1$. Classic examples of coherent systems are $k$-out-of- $n: F$ systems with the structure functions

$$
\phi\left(x_{1}, \ldots, x_{n}\right)=\left\{\begin{array}{ll}
0 & \text { if } n-\sum_{i=1}^{n} x_{i} \geq k, \\
1 & \text { otherwise. }
\end{array} \quad k=1, \ldots, n\right.
$$

It means that the system fails at the moment of the $k$ th failure of its components. A frequently used alternative notion is $k$-out-of- $n: G$ system which works as long as at least $k$ of its components work. Note that the $(n-k+1)$-out-of- $n: G$ system is $k$-out-of- $n: F$. In this paper we prefer to use the $k$-out-of- $n: F$, because it leads to simpler formulae in the description of our results.

Received 3 October 201; revision received 21 February 2014.

* Postal address: Faculty of Mathematics and Computer Sciences, Nicolaus Copernicus University, Chopina 12, 87-100 Torun, Poland.

** Postal address: Institute of Mathematics, Polish Academy of Sciences, Śniadeckich 8, 00-956 Warsaw, Poland. Email address: trychlik@impan.pl 
It is assumed that the lifetimes $X_{1}, \ldots, X_{n}$ of components are random. If the system is composed of identical elements, it is natural to assume that the joint distribution of $X_{1}, \ldots, X_{n}$ is exchangeable. A typical extra assumption that $X_{1}, \ldots, X_{n}$ are independent and identically distributed (i.i.d.) is often not satisfied in practice; for example, failure of some components often causes an increased pressure on the other still working items. If the component lifetimes are exchangeable then the distribution of the semicoherent system lifetime $T$ can be represented as a convex combination of marginal distributions of order statistics $X_{1: n}, \ldots, X_{n: n}$, i.e.

$$
\mathbb{P}(T \leq t)=\sum_{i=1}^{n} s_{i} \mathbb{P}\left(X_{i: n} \leq t\right)
$$

(see Samaniego (1985), (2007), Kochar et al. (1999), and Navarro et al. (2008)). The vector of combination coefficients $s=\left(s_{1}, \ldots, s_{n}\right)$ is called the Samaniego signature and depends only on the system structure. Namely, $s_{i}=\bar{S}_{i-1}-\bar{S}_{i}$, where

$$
\bar{S}_{i}=\frac{1}{\left(\begin{array}{l}
n \\
i
\end{array}\right)} \sum_{x: \sum_{j=1}^{n} x_{j}=n-i} \phi(\boldsymbol{x}), \quad i=0, \ldots, n .
$$

Vectors $\overline{\boldsymbol{S}}=\left(\bar{S}_{0}, \ldots, \bar{S}_{n}\right)$ and $\boldsymbol{S}=\left(1-\bar{S}_{0}, \ldots, 1-\bar{S}_{n}\right)$ are called the tail signature and cumulative signature, respectively, of the system (see Boland (2001) and Gertsbakh et al. (2011)). We also define

$$
\begin{gathered}
\underline{s}_{1}=\min _{1 \leq j \leq n} \frac{1}{j} S_{j}=\min _{1 \leq j \leq n} \frac{1}{j} \sum_{i=1}^{j} s_{i}, \\
\underline{s}_{n}=\max _{1 \leq j \leq n} \frac{1}{j} \bar{S}_{n-j}=\max _{1 \leq j \leq n} \frac{1}{j} \sum_{i=n-j+1}^{n} s_{i}, \\
\bar{s}_{1}=\max _{1 \leq j \leq n} \frac{1}{j} S_{j}=\max _{1 \leq j \leq n} \frac{1}{j} \sum_{i=1}^{j} s_{i}, \\
\bar{s}_{n}=\min _{1 \leq j \leq n} \frac{1}{j} \bar{S}_{n-j}=\min _{1 \leq j \leq n} \frac{1}{j} \sum_{i=n-j+1}^{n} s_{i} .
\end{gathered}
$$

Note that the $k$-out-of- $n: F$ system has lifetime $X_{k: n}$ and signature with coefficients $s_{k}=1$, $s_{i}=0, i \neq k$. Equation (1) implies that the lifetime of a semicoherent system with exchangeable components and signature $s$ has an identical distribution to that of a randomly chosen $k$-out-of- $n: F$ system when the choice probability is $s_{k}$. Using this observation, Boland and Samaniego (2004) introduced a more general and mathematically convenient notion of a mixed system. The mixed system of size $n$ with arbitrary signature $s=\left(s_{1}, \ldots, s_{n}\right)$ such that $0 \leq s_{i} \leq 1$ and $\sum_{i=1}^{n} s_{i}=1$ is randomly chosen among the $k$-out-of- $n: F$ systems (for $k=1, \ldots, n)$ with respective probabilities $s_{1}, \ldots, s_{n}$.

There are known sharp bounds on expectations of system lifetimes with either i.i.d. or exchangeable components under various restrictions on the marginal distributions. They coincide with the bounds on expectations of convex combinations of order statistics. In contrast, much less is known about evaluations of lifetime variances of semicoherent and mixed systems. Sharp upper bounds for lifetime variances of $k$-out-of- $n$ systems with i.i.d. components were derived 
by Papadatos (1995). An extension to the exchangeable case is due to Rychlik (2008). Jasiński et al (2009) presented upper evaluations of variances for arbitrary mixed systems composed of elements with i.i.d. lifetimes. Recently, Miziuła and Rychlik (2014) obtained optimal bounds on lifetime variances of mixed systems composed of exchangeable items in terms of the system signature and the variance of a single component. In this paper this result is generalized by replacing the variance by an arbitrary dispersion measure of a random variable $X$ defined in the following way:

$$
\sigma(X, \rho)=\inf _{\mu \in \mathbb{R}} \mathbb{E} \rho(X-\mu) .
$$

Here $\rho: \mathbb{R} \rightarrow[0, \infty)$ is a function satisfying the following conditions:

(i) $\rho$ is nonincreasing on $(-\infty, 0]$,

(ii) $\rho(0)=0$,

(iii) $\rho$ is nondecreasing on $[0, \infty)$.

In particular, for $\rho(x)=x^{2}$ the dispersion measure is the variance, and for $\rho(x)=|x|$ it becomes the mean absolute deviation from the median. Note, however, that we admit here asymmetric (e.g. popular LINEX) and discontinuous functions $\rho$.

Now we formulate the main result of this paper. It is easily concluded from auxiliary results presented in Section 2. Section 3 contains the proofs.

Theorem 1. Let $X_{1}, \ldots, X_{n}$ be nonnegative, nondegenerate, exchangeable random lifetimes of components of a coherent or mixed reliability system with signature $\boldsymbol{s}=\left(s_{1}, \ldots, s_{n}\right)$ and lifetime denoted by $T$. Let $\rho: \mathbb{R} \rightarrow[0, \infty)$ be a nonincreasing (respectively, nondecreasing) function on $(-\infty, 0]$ (respectively, $[0, \infty)$ ) and vanishing at 0 . If $0<\mathbb{E} \rho\left(X_{1}-\mu\right)<\infty$ for some $\mu \in \mathbb{R}$ then

$$
n \min \left\{\underline{s}_{1}, \bar{s}_{n}\right\} \leq \frac{\sigma(T, \rho)}{\sigma\left(X_{1}, \rho\right)} \leq n \max \left\{\bar{s}_{1}, \underline{s}_{n}\right\} .
$$

Moreover, if $\rho$ is continuous at 0 and $\rho(x)>0$ for $x \neq 0$ then these bounds are the best possible which means that, for an arbitrary system with signature $s=\left(s_{1}, \ldots, s_{n}\right)$, each of two bound in (6), and arbitrary $\varepsilon>0$, there exists an exchangeable joint distribution of nonnegative component lifetimes such that the ratio $\sigma(T, \rho) / \sigma\left(X_{1}, \rho\right)$ is less distant from the bound than $\varepsilon$.

The definitions (2)-(5) imply the following inequalities:

$$
\max \left\{\underline{s}_{1}, \bar{s}_{n}\right\} \leq \frac{1}{n} \sum_{i=1}^{n} s_{i}=\frac{1}{n} \leq \min \left\{\bar{s}_{1}, \underline{s}_{n}\right\} .
$$

Therefore,

$$
0 \leq n \min \left\{\underline{s}_{1}, \bar{s}_{n}\right\} \leq n \max \left\{\underline{s}_{1}, \bar{s}_{n}\right\} \leq 1 \leq n \min \left\{\bar{s}_{1}, \underline{s}_{n}\right\} \leq n \max \left\{\bar{s}_{1}, \underline{s}_{n}\right\} \leq n .
$$

The lower bound is equal to 0 if and only if either $s_{1}=0$ or $s_{n}=0$. This happens for all the coherent systems (see Remark 3, below). The lower bound is equal to 1 if and only if $s_{1}=\cdots=s_{n}=1 / n$. It is the necessary and sufficient condition for the upper bound being equal to 1 as well. The conditions imply that $H=F$ (cf. (8), below), which means that the lifetimes of the system and single component are identical. The upper bound takes on the maximal value $n$ only for the series and parallel systems. We also note that the bounds in (6) are identical for the mutually dual systems; in particular, for the $k$-out-of- $n: F$ and $k$-out-of- $n: G$ ones. 
We prove that the bounds of Theorem 1 are attained in the limit by some exchangeable random variables with identical two-point marginal distributions. This universal, but artificial, construction seems to be not very applicable in reliability theory. Miziuła and Rychlik (2014) showed that the particular variance bounds are attained by Pareto and power marginals and dependence structures resulting in simultaneous failures of some components. We are able to specify more realistic constructions of reliability models providing attainability of the bounds, but they depend on the particular choice of function $\rho$.

\section{Auxiliary results}

Theorem 2. (Rychlik (1993)) Distribution functions $F_{1}, \ldots, F_{n}$ are the distribution functions of consecutive order statistics from an identically distributed sample of size $n$ with a common marginal $F$ if and only if

$$
\begin{gathered}
F_{1} \geq \cdots \geq F_{n}, \\
\sum_{i=1}^{n} F_{i}=n F .
\end{gathered}
$$

The statement is also valid for the narrower class of exchangeable samples.

Theorem 3. (Rychlik (2012)) Let $H=\sum_{i=1}^{n} s_{i} F_{i}$ for some distribution functions satisfying (7) and (8) and arbitrarily fixed $0 \leq s_{i} \leq 1, i=1, \ldots, n$, that sum up to 1 . Let $\underline{S}, \bar{S}:[0,1] \rightarrow$ $[0,1]$ be the greatest convex and smallest concave functions, respectively, satisfying $\underline{S}(0)=$ $\bar{S}(0)=0$ and

$$
\underline{S}\left(\frac{j}{n}\right) \leq \sum_{i=1}^{j} s_{i} \leq \bar{S}\left(\frac{j}{n}\right), \quad j=1, \ldots, n
$$

(see Figure 1). Then

$$
\underline{S}(F(t)) \leq H(t) \leq \bar{S}(F(t))
$$

for all real $t$, and

$$
n \min _{1 \leq i \leq n} s_{i}[F(t)-F(u)] \leq H(t)-H(u) \leq n \max _{1 \leq i \leq n} s_{i}[F(t)-F(u)],
$$

for all real $t>u$.

The inequalities in (10) imply that $H$ is absolutely continuous with respect to $F$, and the corresponding Radon-Nikodym derivative satisfies $n \min _{1 \leq i \leq n} s_{i} \leq \mathrm{d} H / \mathrm{d} F \leq n \max _{1 \leq i \leq n} s_{i} F$ almost surely. Rychlik (1994) proved that conditions (9) and (10) uniquely characterize the lifetime distributions of $k$-out-of- $n$ systems, i.e. they are necessary and sufficient for the specific signatures $s_{k}=\left(e_{k 1}, \ldots, e_{k n}\right), k=1, \ldots, n$, where $e_{k i}=1$ if $i=k$ and 0 otherwise. An extension of the result to slightly more general mixed systems can be found in Rychlik (2012). It is obvious that functions $\underline{S}$ and $\bar{S}$, defined in Theorem 3, are continuous and piecewise linear, and they change their slopes at some points of the form $i / n, 1 \leq i \leq n-1$, only. Therefore, their right-hand side versions of derivatives can be written as

$$
\underline{S}^{\prime}(x)=\sum_{i=1}^{n} n \underline{s}_{i} \mathbf{1}_{[(i-1) / n, i / n)}(x), \quad \bar{S}^{\prime}(x)=\sum_{i=1}^{n} n \bar{s}_{i} \mathbf{1}_{[(i-1) / n, i / n)}(x) .
$$




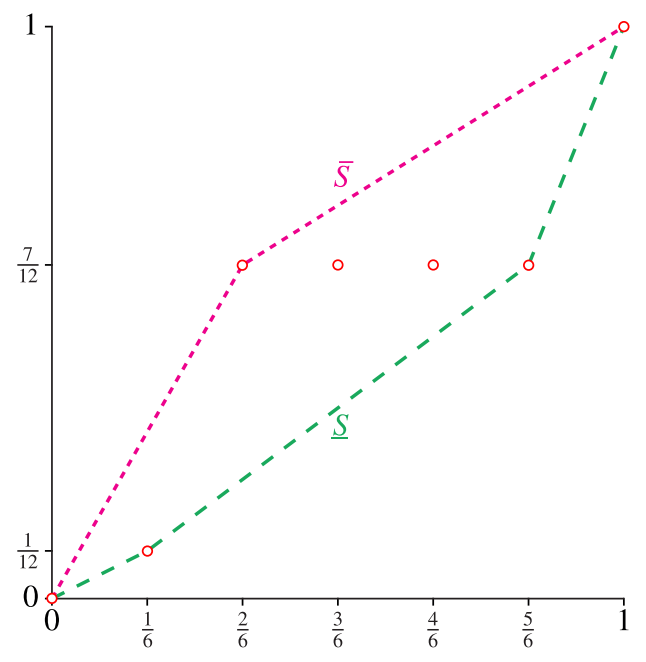

Figure 1: Functions $\underline{S}, \bar{S}$ for $s=\left(\frac{1}{12}, \frac{1}{2}, 0,0,0, \frac{5}{12}\right)$.

Vectors $\underline{s}=\left(\underline{s}_{1}, \ldots, \underline{s}_{n}\right)$ and $\bar{s}=\left(\bar{s}_{1}, \ldots, \bar{s}_{n}\right)$ are the projections of $s=\left(s_{1}, \ldots, s_{n}\right)$ onto the convex cones of nondecreasing and nonincreasing vectors, respectively, in $\mathbb{R}^{n}$ with the standard Euclidean norm. They satisfy $0 \leq \underline{s}_{i}, \bar{s}_{i} \leq 1, i=1, \ldots, n$, and $\sum_{i=1}^{n} \underline{s}_{i}=\sum_{i=1}^{n} \bar{s}_{i}=1$. In particular, $\underline{s}_{1}, \underline{s}_{n}, \bar{s}_{1}$, and $\bar{s}_{n}$ defined here coincide with (2)-(5).

Theorem 4. Suppose that $X_{1}$ has a nondegenerate distribution function $F$, say. Let $\rho: \mathbb{R} \rightarrow$ $[0, \infty)$ be a nonincreasing and nondecreasing function on $(-\infty, 0]$ and $[0, \infty)$, respectively, which satisfies $\rho(0)=0$. If a random variable $T$ has a distribution function $H$ satisfying the assumptions of Theorem 3, then

$$
n \min \left\{\underline{s}_{1}, \bar{s}_{n}\right\} \leq \frac{\mathbb{E} \rho(T-\mu)}{\mathbb{E} \rho\left(X_{1}-\mu\right)} \leq n \max \left\{\bar{s}_{1}, \underline{s}_{n}\right\}
$$

for all $\mu \in \mathbb{R}$ such that $0<\mathbb{E} \rho\left(X_{1}-\mu\right)<\infty$, where $\underline{s}_{1}, \underline{s}_{n}, \bar{s}_{1}$, and $\bar{s}_{n}$ are defined in (2)-(5).

Theorem 5. Under the assumptions of Theorem 4, the inequalities in (6) hold. Moreover, if $\rho$ is continuous at 0 and positive for all nonzero arguments, then the bounds (6) are attained in the limit. Precisely, for every signature sequence $\left(s_{1}, \ldots, s_{n}\right)$, function $\rho, \varepsilon>0$, and each of two bounds in (6), there is an n-dimensional distribution with marginals $F_{1} \geq \cdots \geq F_{n}$ such that, for random variables $X_{1}$ and $T$ with distribution functions $F=(1 / n) \sum_{i=1}^{n} F_{i}$ and $H=\sum_{i=1}^{n} s_{i} F_{i}$, respectively, the ratio $\sigma(T, \rho) / \sigma\left(X_{1}, \rho\right)$ is less distant from the respective bound than $\varepsilon$.

Theorems 4 and 5 are proved in Section 3. In view of Theorem 2, Theorem 5 immediately implies Theorem 1. Note that we can admit that the random variables $X_{1}, \ldots, X_{n}$, defined in Theorem 1, do not need to be exchangeable and can take on some negative values as well. Actually, we provide here optimal bounds for dispersions of order statistics $X_{I: n}$ based on an arbitrary identically distributed sequence $X_{1}, \ldots, X_{n}$ with randomly chosen index $I$ which is independent of the sequence and has probability distribution $\mathbb{P}(I=i)=s_{i}, i=1, \ldots, n$. The randomly selected order statistics $X_{I: n}$ were applied in optimal nonparametric quantile 
estimation by Uhlmann (1963) and Zieliński (1999), (2001). We finally observe that Theorem 1 holds for the $k$-out-of- $n: F$ system when the exchangeability assumption is dropped and we merely require that the component lifetimes have identical marginal distributions.

\section{Proofs}

Using Theorem 3 we can check that if $\mathbb{E} \rho\left(X_{1}-\mu\right)<\infty$ for some $\mu \in \mathbb{R}$ then

$$
\begin{aligned}
\mathbb{E} \rho(T-\mu) & =\int \rho(x-\mu) \frac{\mathrm{d} H}{\mathrm{~d} F}(x) F(\mathrm{~d} x) \\
& \leq n \max _{1 \leq i \leq n} s_{i} \int \rho(x-\mu) F(\mathrm{~d} x) \\
& =n \max _{1 \leq i \leq n} s_{i} \mathbb{E} \rho\left(X_{1}-\mu\right) \\
& <+\infty
\end{aligned}
$$

\subsection{Proof of the first inequality in Theorem 4}

To prove that the first (i.e. the left-hand side) inequality in Theorem 4 holds, we proceed as follows. If either $\underline{s}_{1}=0$ or $\bar{s}_{n}=0$ then the inequality is obvious. So we assume that $\underline{s}_{1}>0$ and $\bar{s}_{n}>0$. Set $0<m=n \min \left\{\underline{s}_{1}, \bar{s}_{n}\right\} \leq 1$, and define functions $\underline{r}, \bar{r}:[0,1] \rightarrow[0,1]$ as

$$
\underline{r}(x)=\left\{\begin{array}{ll}
m x, & x<1, \\
1, & x=1,
\end{array} \quad \bar{r}(x)= \begin{cases}0, & x=0, \\
m(x-1)+1, & x>0\end{cases}\right.
$$

(see Figure 2). Since $\underline{r} \leq \underline{S}$ and $\bar{r} \geq \bar{S}$, by Theorem 3, we have

$$
\underline{r} \circ F \leq H \leq \bar{r} \circ F \text {. }
$$

Define

$$
G_{\mu}(x)=m F(x)+(1-m) \mathbf{1}_{[\mu, \infty)}(x)= \begin{cases}\underline{r} \circ F(x), & x<\mu, \\ \bar{r} \circ F(x), & x \geq \mu\end{cases}
$$

(see Figure 2). We have $H(x) \geq G_{\mu}(x)$ for $x<\mu$ and $H(x) \leq G_{\mu}(x)$ for $x \geq \mu$. Therefore,

$$
\int \rho(x-\mu) G_{\mu}(\mathrm{d} x) \leq \int \rho(x-\mu) H(\mathrm{~d} x)=\mathbb{E} \rho(T-\mu) .
$$

Moreover,

$$
\begin{aligned}
\int \rho(x-\mu) G_{\mu}(\mathrm{d} x) & =m \int \rho(x-\mu) F(\mathrm{~d} x)+(1-m) \int \rho(x-\mu) \mathbf{1}_{[\mu, \infty)}(\mathrm{d} x) \\
& =m \int \rho(x-\mu) F(\mathrm{~d} x)+(1-m) \rho(0) \\
& =m \mathbb{E} \rho\left(X_{1}-\mu\right) .
\end{aligned}
$$

The inequality is proved.

Remark 1. It is easy to see from (12) and (13) that the condition $\mathbb{E} \rho(T-\mu)<\infty$ for fixed $\mu \in \mathbb{R}$ implies $\mathbb{E} \rho\left(X_{1}-\mu\right)<\infty$ as well. Combining this result with (11) we conclude that

$$
\mathbb{E} \rho\left(X_{1}-\mu\right)<\infty \quad \Longleftrightarrow \quad \mathbb{E} \rho(T-\mu)<\infty .
$$




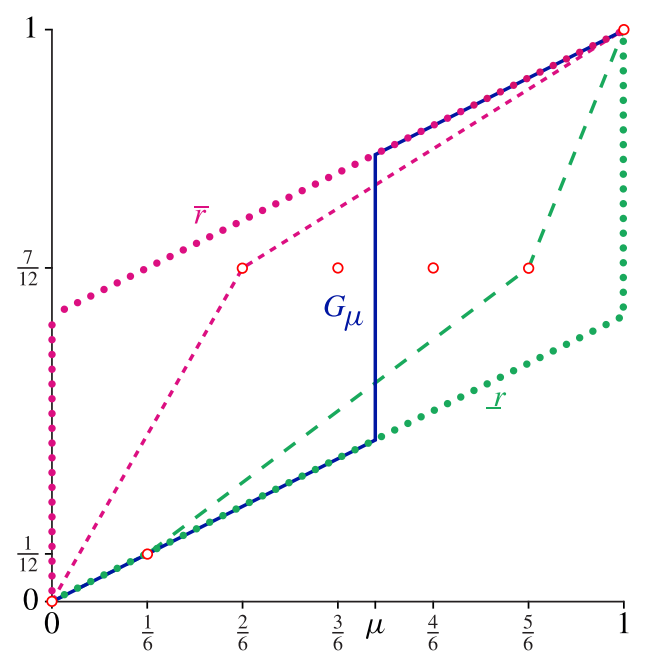

FiguRE 2: Functions $\underline{r}, \bar{r}$, and $G_{\mu}$ for $s=\left(\frac{1}{12}, \frac{1}{2}, 0,0,0, \frac{5}{12}\right)$ and $F \sim \mathrm{U}(0,1)$.

\subsection{Proof of the second inequality in Theorem 4}

To prove that the second (i.e. the right-hand side) inequality in Theorem 4 holds, we proceed as follows. Define $1 \leq M=n \max \left\{\bar{s}_{1}, \underline{s}_{n}\right\} \leq n$, and define the functions $\underline{R}, \bar{R}:[0,1] \rightarrow[0,1]$ by

$$
\underline{R}(x)=\max \{M(x-1)+1,0\}, \quad \bar{R}(x)=\min \{M x, 1\}
$$

(see Figure 3). Since $\underline{R} \leq \underline{S}$ and $\bar{R} \geq \bar{S}$, Theorem 3 implies that

$$
\underline{R} \circ F \leq H \leq \bar{R} \circ F \text {. }
$$

Assume that $H(\mu)=\alpha \in[0,1]$. Let

$$
C=\left\{x \in \mathbb{R}: \frac{\alpha}{M} \leq F(x) \leq 1-\frac{1-\alpha}{M}\right\} .
$$

It is clear that $\mu \in C$. Define

$$
\begin{aligned}
G_{\alpha}(x) & = \begin{cases}M F(x), & F(x)<\alpha / M, \\
\alpha, & x \in C, \\
M(F(x)-1)+1, & F(x)>1-(1-\alpha) / M\end{cases} \\
& = \begin{cases}\bar{R} \circ F(x), & F(x)<\alpha / M, \\
\alpha, & x \in C, \\
\underline{R} \circ F(x), & F(x)>1-(1-\alpha) / M\end{cases}
\end{aligned}
$$

(see Figure 3). Such a $G_{\alpha}$ is a distribution function. We have $H(x) \leq G_{\alpha}(x)$ for $x<\mu$ and $H(x) \geq G_{\alpha}(x)$ for $x \geq \mu$. Therefore,

$$
\mathbb{E} \rho(T-\mu)=\int \rho(x-\mu) H(\mathrm{~d} x) \leq \int \rho(x-\mu) G_{\alpha}(\mathrm{d} x) .
$$




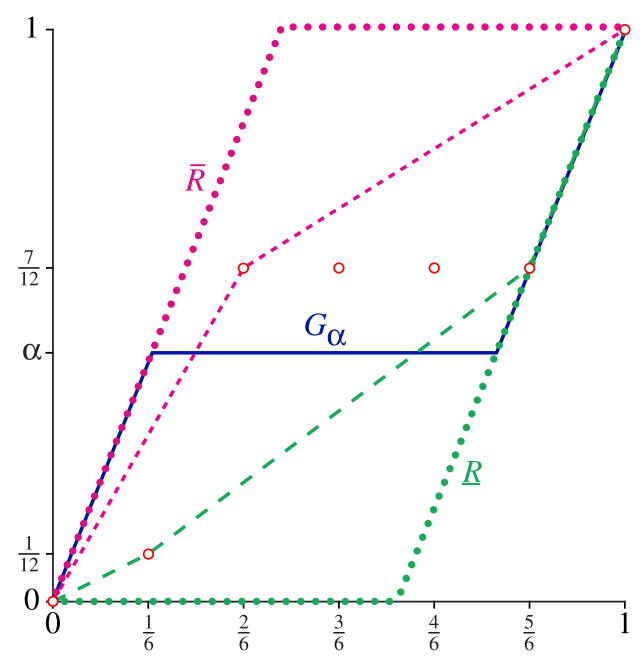

FIGURE 3: Functions $\underline{R}, \bar{R}$, and $G_{\alpha}$ for $s=\left(\frac{1}{12}, \frac{1}{2}, 0,0,0, \frac{5}{12}\right)$ and $F \sim \mathrm{U}(0,1)$.

Moreover,

$\int \rho(x-\mu) G_{\alpha}(\mathrm{d} x)=M \int_{\mathbb{R} \backslash C} \rho(x-\mu) F(\mathrm{~d} x) \leq M \int \rho(x-\mu) F(\mathrm{~d} x)=M \mathbb{E} \rho\left(X_{1}-\mu\right)$.

The proof is complete.

\subsection{Proof of the inequalities in Theorem 5}

If either $\underline{s}_{1}=0$ or $\bar{s}_{n}=0$ then the left-hand side inequality in (6) is obvious. So we assume that $\underline{s}_{1}>0, \bar{s}_{n}>0$, and $0<m=n \min \left\{\underline{s}_{1}, \bar{s}_{n}\right\} \leq 1$. There exists a sequence $\mu_{k}$, $k=1,2, \ldots$, where

$$
\mathbb{E} \rho\left(T-\mu_{k}\right) \searrow \inf _{\mu \in \mathbb{R}} \mathbb{E} \rho(T-\mu)=\sigma(T, \rho)
$$

as $k \rightarrow \infty$. For this sequence, by Theorem 4 and Remark 1 , we also have

$$
\sigma\left(X_{1}, \rho\right) \leq \mathbb{E} \rho\left(X_{1}-\mu_{k}\right) \leq \frac{1}{m} \mathbb{E} \rho\left(T-\mu_{k}\right) \searrow \frac{1}{m} \sigma(T, \rho)
$$

and, consequently,

$$
m \sigma\left(X_{1}, \rho\right) \leq \sigma(T, \rho) .
$$

Now take $1 \leq M=n \max \left\{\bar{s}_{1}, \underline{s}_{n}\right\} \leq n$. There exists a sequence $\mu_{k}, k=1,2, \ldots$, where

$$
\mathbb{E} \rho\left(X_{1}-\mu_{k}\right) \searrow \inf _{\mu \in \mathbb{R}} \mathbb{E} \rho\left(X_{1}-\mu\right)=\sigma\left(X_{1}, \rho\right)
$$

as $k \rightarrow \infty$. From Theorem 4, we obtain

$$
\sigma(T, \rho) \leq \mathbb{E} \rho\left(T-\mu_{k}\right) \leq M \mathbb{E} \rho\left(X_{1}-\mu_{k}\right) \searrow M \sigma\left(X_{1}, \rho\right),
$$

which allows us to conclude that

$$
\sigma(T, \rho) \leq M \sigma\left(X_{1}, \rho\right)
$$




\subsection{Proof of the optimality of Theorem 5}

The proof is based on two constructions. For an arbitrarily chosen $j \in\{1, \ldots, n\}$ we first define exchangeable random variables $X_{1}, \ldots, X_{n}$ with a joint distribution dependent on the parameter $\alpha \in(0,1)$ and a variable $T$ whose distribution is the convex combination of marginal distributions of $X_{1: n}, \ldots, X_{n: n}$ with coefficients $s_{1}, \ldots, s_{n}$, so that

$$
\lim _{\alpha \searrow 0} \frac{\sigma(T, \rho)}{\sigma\left(X_{1}, \rho\right)}=\frac{n}{j} \sum_{i=1}^{j} s_{i} .
$$

Another construction leads to the relation

$$
\lim _{\alpha \searrow 0} \frac{\sigma(T, \rho)}{\sigma\left(X_{1}, \rho\right)}=\frac{n}{j} \sum_{i=n-j+1}^{n} s_{i}
$$

Since

$$
\left\{\underline{s}_{1}, \bar{s}_{1}\right\} \subset\left\{\frac{1}{j} \sum_{i=1}^{j} s_{i}: j=1, \ldots, n\right\}
$$

and

$$
\left\{\underline{s}_{n}, \bar{s}_{n}\right\} \subset\left\{\frac{1}{j} \sum_{i=n-j+1}^{n} s_{i}: j=1, \ldots, n\right\},
$$

proving (14) and (15) is sufficient for concluding sharpness of bounds in Theorem 5.

First, we describe a model providing equality (14). Choose $c \in(0, \infty)$ such that $\rho$ is continuous at $c$ and $-c$. Fix $j \in\{1, \ldots, n\}$. Suppose that we have two urns containing $n$ balls with values

$$
(\underbrace{0, \ldots, 0}_{j}, \underbrace{c, \ldots, c}_{n-j}), \quad(\underbrace{c, \ldots, c}_{n}) .
$$

Let $\alpha \in(0,1)$. First we choose either the first or the second urn with probability $\alpha$ and $1-\alpha$, respectively. Then we draw all the balls from the chosen urn without replacement. Let $X_{1}, \ldots, X_{n}$ denote the values of consecutively drawn balls. These random variables are exchangeable and have the two-point marginal distribution

$$
\mathbb{P}_{\alpha}\left(X_{1}=0\right)=\alpha \frac{j}{n}=1-\mathbb{P}_{\alpha}\left(X_{1}=c\right)
$$

dependent on $\alpha$. It is also easy to see that

$$
\mathbb{P}_{\alpha}\left(X_{i: n}=0\right)=1-\mathbb{P}_{\alpha}\left(X_{i: n}=c\right)= \begin{cases}\alpha, & i \leq j, \\ 0, & i>j\end{cases}
$$

for $i=1, \ldots, n$. Now we obtain a random variable $T$ by choosing $X_{i: n}$ with probability $s_{i}$. It has distribution

$$
\mathbb{P}_{\alpha}(T=0)=\alpha \sum_{i=1}^{j} s_{i}=1-\mathbb{P}_{\alpha}(T=c) .
$$

We aim at proving that the construction allows us to get (14). 
We have

$$
\begin{gathered}
\mathbb{E}_{\alpha} \rho\left(X_{1}-x\right)=\alpha \frac{j}{n} \rho(-x)+\left(1-\alpha \frac{j}{n}\right) \rho(c-x), \\
\mathbb{E}_{\alpha} \rho(T-y)=\alpha \sum_{i=1}^{j} s_{i} \rho(-y)+\left(1-\alpha \sum_{i=1}^{j} s_{i}\right) \rho(c-y) .
\end{gathered}
$$

Relations $\rho(-x) \geq \rho(-c)$ and $\rho(c-x)>\rho(0)$ for $x>c$ imply

$$
\mathbb{E}_{\alpha} \rho\left(X_{1}-x\right)>\mathbb{E}_{\alpha} \rho\left(X_{1}-c\right), \quad x>c .
$$

Similarly,

$$
\mathbb{E}_{\alpha} \rho\left(X_{1}-x\right)<\mathbb{E}_{\alpha} \rho\left(X_{1}-0\right), \quad x<0 .
$$

Let $x_{\alpha} \in \mathbb{R}$ satisfy

$$
\mathbb{E}_{\alpha} \rho\left(X_{1}-x_{\alpha}\right) \leq \sigma_{\alpha}\left(X_{1}, \rho\right)+\alpha^{2} .
$$

By (16) and (17), we can assume that $x_{\alpha} \in[0, c]$. Using the same arguments, we can find $y_{\alpha} \in[0, c]$ such that

$$
\mathbb{E}_{\alpha} \rho\left(T-y_{\alpha}\right) \leq \sigma_{\alpha}(T, \rho)+\alpha^{2}
$$

So we have

$$
\frac{\sigma_{\alpha}(T, \rho)}{\sigma_{\alpha}\left(X_{1}, \rho\right)} \geq \frac{\mathbb{E}_{\alpha} \rho\left(T-y_{\alpha}\right)-\alpha^{2}}{\mathbb{E}_{\alpha} \rho\left(X_{1}-x_{\alpha}\right)}
$$

and

It suffices to prove that

$$
\frac{\sigma_{\alpha}\left(X_{1}, \rho\right)}{\sigma_{\alpha}(T, \rho)} \geq \frac{\mathbb{E}_{\alpha} \rho\left(X_{1}-x_{\alpha}\right)-\alpha^{2}}{\mathbb{E}_{\alpha} \rho\left(T-y_{\alpha}\right)} .
$$

$$
\lim _{\alpha \searrow 0} \frac{\mathbb{E}_{\alpha} \rho\left(T-y_{\alpha}\right)-\alpha^{2}}{\mathbb{E}_{\alpha} \rho\left(X_{1}-x_{\alpha}\right)}=\frac{n}{j} \sum_{i=1}^{j} s_{i}
$$

and

$$
\lim _{\alpha \searrow 0} \frac{\mathbb{E}_{\alpha} \rho\left(X-x_{\alpha}\right)-\alpha^{2}}{\mathbb{E}_{\alpha} \rho\left(T-y_{\alpha}\right)}=\left(\frac{n}{j} \sum_{i=1}^{j} s_{i}\right)^{-1} .
$$

We will show that (18) holds; the proof of (19) is fully analogous. We need the following facts:

(i) $\lim _{\alpha \searrow 0} x_{\alpha}=c$ and $\lim _{\alpha \searrow 0} y_{\alpha}=c$,

(ii) $\lim _{\alpha \searrow 0} \rho\left(c-x_{\alpha}\right) / \alpha=0$ and $\lim _{\alpha \searrow 0} \rho\left(c-y_{\alpha}\right) / \alpha=0$.

We now prove that these facts hold.

(i) Suppose that there exist $\varepsilon>0$ and $\left(\alpha_{k}\right)_{k=1}^{\infty}$ such that $\alpha_{k} \rightarrow 0$ and $x_{\alpha_{k}} \leq c-\varepsilon$. Then

$$
\begin{aligned}
\mathbb{E}_{\alpha_{k}} \rho\left(X_{1}-x_{\alpha_{k}}\right) & =\alpha_{k} \frac{j}{n} \rho\left(-x_{\alpha_{k}}\right)+\left(1-\alpha_{k} \frac{j}{n}\right) \rho\left(c-x_{\alpha_{k}}\right) \\
& \geq\left(1-\alpha_{k} \frac{j}{n}\right) \rho(\varepsilon) \\
& \rightarrow \rho(\varepsilon) \\
& >0
\end{aligned}
$$


whereas,

$$
\mathbb{E}_{\alpha_{k}} \rho\left(X_{1}-x_{\alpha_{k}}\right) \leq \sigma_{\alpha_{k}}\left(X_{1}, \rho\right)+\alpha_{k}^{2} \leq \mathbb{E}_{\alpha_{k}} \rho\left(X_{1}-c\right)+\alpha_{k}^{2}=\alpha_{k} \frac{j}{n} \rho(-c)+\alpha_{k}^{2} \rightarrow 0 .
$$

So we get the contradiction. Analogously, we check that $\lim _{\alpha \searrow 0} y_{\alpha}=c$.

(ii) We have

$$
\begin{aligned}
1 & \geq \frac{\sigma_{\alpha}\left(X_{1}, \rho\right)}{\mathbb{E}_{\alpha} \rho\left(X_{1}-c\right)} \\
& \geq \frac{\mathbb{E}_{\alpha} \rho\left(X_{1}-x_{\alpha}\right)-\alpha^{2}}{\mathbb{E}_{\alpha} \rho\left(X_{1}-c\right)} \\
& =\frac{\alpha(j / n) \rho\left(-x_{\alpha}\right)+(1-\alpha j / n) \rho\left(c-x_{\alpha}\right)-\alpha^{2}}{\alpha(j / n) \rho(-c)} .
\end{aligned}
$$

Hence,

$$
0 \leq \frac{\rho\left(c-x_{\alpha}\right)}{\alpha} \leq \frac{(j / n)\left[\rho(-c)-\rho\left(-x_{\alpha}\right)\right]+\alpha}{1-\alpha j / n} \rightarrow 0
$$

as $\alpha \searrow 0$. Analyzing $\rho\left(c-y_{\alpha}\right) / \alpha$ we proceed analogously.

Now, using these facts, we can see that

$$
\frac{\mathbb{E}_{\alpha} \rho\left(T-y_{\alpha}\right)}{\mathbb{E}_{\alpha} \rho\left(X_{1}-x_{\alpha}\right)}=\frac{\sum_{i=1}^{j} s_{i}\left[\rho\left(-y_{\alpha}\right)-\rho\left(c-y_{\alpha}\right)\right]+\rho\left(c-y_{\alpha}\right) / \alpha}{(j / n)\left[\rho\left(-x_{\alpha}\right)-\rho\left(c-x_{\alpha}\right)\right]+\rho\left(c-x_{\alpha}\right) / \alpha} \rightarrow \frac{n}{j} \sum_{i=1}^{j} s_{i}
$$

and

$$
\frac{\alpha^{2}}{\mathbb{E}_{\alpha} \rho\left(X_{1}-x_{\alpha}\right)}=\frac{\alpha^{2}}{\alpha(j / n) \rho\left(-x_{\alpha}\right)+(1-\alpha j / n) \rho\left(c-x_{\alpha}\right)} \leq \frac{\alpha}{(j / n) \rho(-c / 2)} \rightarrow 0,
$$

which completes the proof of (18). This together with (19) implies (14).

Similar calculations allow us to prove that (15) is attained by the analogous model of two urns containing balls with the following values:

$$
(\underbrace{c, \ldots, c}_{j}, \underbrace{0, \ldots, 0}_{n-j}), \quad(\underbrace{0, \ldots, 0}_{n})
$$

when the probability $\alpha$ of choosing the first urn tends to 0 .

Remark 2. In the above proof we applied an urn model of elementary probability theory. In the reliability setup we could consider $n$ system components with two possible failure schemes. Either $j$ randomly chosen components fail at a fixed time $t$, say, and the other $n-j$ do so $c$ time units later, or all the components fail simultaneously at the moment $t+c$. The probabilities of these two cases are $\alpha$ and $1-\alpha$, respectively. This model yields (14). Relation (15) can be derived once we interchange the failure times $t$ and $t+c$ in the previous construction.

Remark 3. If a coherent system has at least two elements, then either $s_{1}=0$ or $s_{n}=0$. We prove this claim using the definition of a coherent system. Suppose that $n \geq 2$ and $s_{1}, s_{n}>0$. The condition $s_{n}>0$ implies that there exists a sequence of components $\left(x_{1}, \ldots, x_{n}\right) \in\{0,1\}^{n}$ containing $n-1$ zeros such that $\phi\left(x_{1}, \ldots, x_{n}\right)=1$. Without loss of generality we can write $\phi(1,0, \ldots, 0)=1$. On the other hand, $s_{1}>0$ assures that $\phi\left(x_{1}, \ldots, x_{n}\right)=0$ for some 
$\left(x_{1}, \ldots, x_{n}\right) \in\{0,1\}^{n}$ such that $\sum_{i=1}^{n} x_{i}=n-1$. Since $\phi\left(x_{1}, \ldots, x_{n}\right)=1$ if $x_{1}=1$, it must be the case that $x_{1}=0$ when $\phi\left(x_{1}, \ldots, x_{n}\right)=0$. Thus, the state vector $\left(x_{1}, \ldots, x_{n}\right)$ with one zero element for which $\phi\left(x_{1}, \ldots, x_{n}\right)=0$ must be the vector $(0,1, \ldots, 1)$. But this implies that all components except for the first are irrelevant. This contradicts the assumption that the system is coherent.

\section{Acknowledgements}

The authors would like to thank an anonymous referee for helpful comments which allowed them to correct mistakes and improve the presentation of the paper. The second author was supported by the Polish Ministry of Science and Higher Education (grant number N N201 416739).

\section{References}

Barlow, R. E. And Proschan, F. (1965). Mathematical Theory of Reliability. John Wiley, New York.

Barlow, R. E. And Proschan, F. (1975). Statistical Theory of Reliability and Life Testing. Holt, Rinehart and Winston, New York.

Boland, P. J. (2001). Signatures of indirect majority systems. J. Appl. Prob. 38, 597-603.

Boland, P. J. And SAmaniego, F. J. (2004). The signature of a coherent system and its applications in reliability. In Mathematical Reliability: An Expository Perspective (Internat. Ser. Operat. Res. Manag. Sci. 67), Kluwer, Boston, MA, pp. 3-30.

Gertsbakh, I., ShPungin, Y. And Spizzichino, F. (2011). Signatures of coherent systems built with separate modules. J. Appl. Prob. 48, 843-855.

JASIŃSKi, K., NAVARRO, J. AND RYCHLIK, T. (2009). Bounds on variances of lifetimes of coherent and mixed systems. J. Appl. Prob. 46, 894-908.

Kochar, S., Mukerjee, H. and Samaniego, F. J. (1999). The 'signature' of a coherent system and its application to comparisons among systems. Naval Res. Logistics 46, 507-523.

MiziuŁa, P. AND RYCHLIK, T. (2014). Sharp bounds for lifetime variances of reliability systems with exchangeable components. IEEE Trans. Reliab. 63, 850-857.

Navarro, J., Samaniego, F. J., Balakrishnan, N. and Bhattacharya, D. (2008). On the application and extension of system signatures in engineering reliability. Naval Res. Logistics 55, 313-327.

Papadatos, N. (1995). Maximum variance of order statistics. Ann. Inst. Statist. Math. 47, 185-193.

Rychlik, T. (1993). Bounds for expectation of $L$-estimates for dependent samples. Statistics 24, 1-7.

Rychlik, T. (1994). Distributions and expectations of order statistics for possibly dependent random variables. J. Multivariate Anal. 48, 31-42.

Rychlik, T. (2008). Extreme variances of order statistics in dependent samples. Statist. Prob. Lett. 78, $1577-1582$.

Rychlik, T. (2012). Applications of Samaniego signatures to bounds on variances of coherent and mixed system lifetimes. In Recent Advances in System Reliability, Springer, London, pp. 63-77.

SAmaniego, F. J. (1985). On closure of the IFR class under formation of coherent systems. IEEE Trans. Reliab. 34, 69-72.

Samaniego, F. J. (2007). System Signatures and their Applications in Engineering Reliability (Internat. Ser. Operat. Res. Manag. Sci. 110). Springer, New York.

Uhlmann, W. (1963). Ranggrössen als Schätzfunktionen. Metrika 7, 23-40.

ZIELIŃsKi, R. (1999). Best equivariant nonparametric estimator of a quantile. Statist. Prob. Lett. 45, 79-84.

ZIELIŃSKI, R. (2001). PMC-optimal nonparametric quanitle esitmator. Statistics 35, 453-462. 